\title{
DASS21: a useful tool in the psychological profile evaluation of dialysis patients
}

\begin{abstract}
Background: The effect of dialysis treatment is complex, with both clinical and sociopsychological effects. In this study, we aimed to assess the psychological status of this growing population of end-stage renal disease. Methods: Using the Short Form of Depression, Anxiety and Stress Scale (DASS21) questionnaire, we aimed (1) to measure the psychological states of hemodialysis (HD) or peritoneal dialysis (PD) subjects from 15 sites, (2) to compare DASS21 scores between HD and PD, and (3) to identify the associated demographic and medical factors of better psychological states. Results: A total of 1,332 were eligible for analysis. Stress (48\%) recorded the highest negative emotional states, followed by depression (37\%) and anxiety (20\%). By multivariate analysis, normal body mass index weight status, religion and absence of coronary artery disease were associated with lower score for depression, anxiety and stress, respectively. Tertiary education was associated with the lowest score in depression and anxiety, whereas HD had a lower score in stress than PD. A younger age was associated with worse DASS21 score of anxiety and stress. Conclusions: Obesity, religion and coronary artery disease were significantly associated with all 3 symptoms of depression, anxiety and stress. Older age has a protective effect on anxiety and stress. Further study is needed to evaluate the relationship between these significant factors and each psychological state.
\end{abstract}

Keyword: DASS; Depression; Anxiety; Stress; Dialysis 